\title{
Research Article \\ Efficacy of Platelet-Rich Plasma in Retarding Intervertebral Disc Degeneration: A Meta-Analysis of Animal Studies
}

\author{
Pei Li, ${ }^{1}$ Ruijie Zhang, ${ }^{2}$ and Qiang Zhou ${ }^{1}$ \\ ${ }^{1}$ Department of Orthopedic Surgery, Southwest Hospital, Third Military Medical University, Chongqing 400038, China \\ ${ }^{2}$ Department of Respiratory Medicine, The Third Xiangya Hospital, Central South University, Changsha, Hunan 410013, China \\ Correspondence should be addressed to Qiang Zhou; zq_tlh@163.com
}

Received 7 January 2017; Revised 24 March 2017; Accepted 11 April 2017; Published 2 July 2017

Academic Editor: Giuseppe Filardo

Copyright ( 2017 Pei Li et al. This is an open access article distributed under the Creative Commons Attribution License, which permits unrestricted use, distribution, and reproduction in any medium, provided the original work is properly cited.

\begin{abstract}
Objectives. Several animal studies have demonstrated the positive effects of platelet-rich plasma (PRP) on disc degeneration retardation. The present meta-analysis was to verify the efficacy of PRP in retarding disc degeneration in animal. Methods. Relevant studies were identified and evaluated according to our inclusion and exclusion criteria. The standardized mean difference (SMD) and related 95\% confidence interval (95\% CI) were estimated to assess PRP efficiency. Results. In total, eleven studies were included in this meta-analysis. Significant differences were found in the PRP treatment group, which showed increased disc height (SMD $=2.66,95 \%$ CI: $1.86,3.47, p=0.000)$, increased MRI T2 signal intensity (SMD $=-3.29,95 \% \mathrm{CI}:-4.44,-2.13, p=0.000)$, and decreased histological degeneration grade ( $\mathrm{SMD}=-4.28,95 \% \mathrm{CI}:-5.26,-3.30, p=0.000)$. However, no significant increase in collagen II expression was found (SMD $=25389.74,95 \%$ CI: $-27585.72,78365.21, p=0.348$ ). Apart from the subgroup analysis of the disc height based on animal species (pig) and disc degeneration model (chymopapain induction), other subgroup analysis based on animal species (rabbit and rat), study design, disc degeneration model, and follow-up period demonstrated that PRP treatment can significantly restore disc height and increase MRI T2 signal intensity. Conclusions. PRP treatment is potentially effective in restoring disc height of rodent rabbit and rat, reducing histological degeneration grade, and increasing MRI T2 image signal. PRP injection may be promising therapy for retarding disc degeneration.
\end{abstract}

\section{Introduction}

Intervertebral disc degeneration (IDD) is a main contributor to low back pain (LBP) which leads to tremendous medical expenditure [1-7]. No effective treatment for IDD currently exists to reverse and repair disc degeneration. During recent decades, finding an effective treatment for and identifying the molecular biological mechanism of IDD become research priorities.

Anatomically, the intervertebral disc (IVD) consists of the central highly hydrated nucleus pulposus (NP), the peripheral lamellae annulus fibrosus (AF), and the superior and inferior cartilage endplates (CEPs) [8]. Physiologically, the swelling pressure within the NP region caused by the negatively charged proteoglycans facilitates spinal load absorption [9]. During IDD, the disc undergoes complex biochemical and cellular changes including loss of proteoglycan content, the transition of type II collagen to type I collagen, and decreases in NP cell density [10-12]. These degenerative changes directly lead to attenuated mechanical function of the IVD and ultimately induce structural disruptions, such as AF tears and NP protrusion.

IVD has a limited ability to self-repair, and the degenerative process usually progresses until it is irreversible [13]. Therefore, developing effective treatments for disc degeneration has become imperative. Previously, growth factors, such as transforming growth factor (TGF)- $\beta 1$ [14-16], bone morphogenetic protein (BMP)-7 [17-19], growth differentiation factor-5 [20], insulin-like growth factor (IGF)-1 [17, 21, 22], basic fibroblast growth factor [23] and platelet-derived growth factor (PDGF) [23-25], were adopted to restore matrix synthesis and disc cell viability in vivo or in vitro. However, these recombinant growth factors have certain practical disadvantages, and few are available in clinical practice [26].

Platelet-rich plasma (PRP) is easily collected from the peripheral blood. It is a growth factor cocktail that contains 
numerous growth factors, including TGF- $\beta 1$, IGF-1, VEGF, EGF, FGF, HGF, and PDGF [27]. Several cell culture studies have demonstrated that PRP could effectively promote disc cell proliferation and enhance extracellular matrix synthesis [28-32]. Furthermore, some in vivo animal studies have demonstrated that PRP injection into a degenerated disc could restore disc height, increase NP hydration, and upregulate the expression of matrix macromolecules [26, 29, 33-42].

Though multiple animal studies have investigated the efficacy of PRP treatment in retarding disc degeneration, single studies often have limited sample size, and the experimental designs differ across studies. Because a consensus on the efficacy of PRP treatment in disc regeneration in animal models is a prerequisite for human clinical trials, we performed this meta-analysis to evaluate the efficacy of PRP treatment in retarding disc degeneration. This metaanalysis focused on the following outcomes: alterations in disc height, NP hydration as indicated by MRI T2 signal intensity, histological disc degeneration grade, and collagen II expression.

\section{Materials and Methods}

2.1. Statement of Our Reporting Standard. This meta-analysis complies with the Preferred Reporting Items for Systematic Reviews and Meta-Analyses (PRISMA) statement [43].

2.2. Search Strategy. A comprehensive literature search was performed using the following databases: MEDLINE, PubMed, EMBASE, the Cochrane Library, and the China National Knowledge Internet (CNKI). The last search date was October 16, 2016. English and Chinese papers were included in the present study. The search terms were "plateletrich plasma/PRP" and "intervertebral disc/intervertebral disk". To identify other potentially relevant studies, authors' bibliographies and the reference lists of each identified study were manually retrieved.

2.3. Inclusion and Exclusion Criteria. First, the title and abstract of each article were evaluated to eliminate irrelevant or reduplicative literature. The full text of the remaining papers was then screened in accordance with our inclusion and exclusion criteria. In this meta-analysis, we included preclinical controlled trials (randomized (RCT) or nonrandomized (N-RCT)) that investigated the efficacy of PRP treatment in disc regeneration. We focused on the following outcomes at the final follow-up time point: disc height alteration, MRI T2 signal intensity, histological disc degeneration grade, and collagen II expression. Any type of disc degeneration model, induced by needle puncture, mechanical loading, or chemonucleolysis was included regardless of the animal species. Similarly, we did not limit the mode of intervention in the control group. We excluded studies without the original data (e.g., reviews, letters, or editorials) and studies without the outcomes of interest.

2.4. Data Extraction. Data extraction from the eligible studies was performed by two independent reviewers. Disagreements between the reviewers were resolved after discussion, and consensus was eventually reached on all extracted information. The extracted information included the first author's name, publication year, animal species, disc degeneration model (traumatic or chemical assault), interventions in the control group, and the outcomes of interest. Additionally, when the outcomes of interest were analyzed at more than one time point, the data from the final time point was used. When a study contained multiple experimental groups, the PRP treatment group and the related control group were selected. MRI T2 signal intensity was classified using the Pfirrmann classification, with grades I to $\mathrm{V}$ being scored from 1 to 5 [44]. MRI T2 signal intensity determined using the Thompson classification was reclassified using the Pfirrmann classification by two reviewers. Histological degeneration grade was classified using the grading scale described by Nomura et al. [45], with grades 0 to 5 being scored from 0 to 5 .

2.5. Quality Assessment. The methodological quality of the eligible studies was assessed according to the Collaborative Approach to Meta-Analysis and Review of Animal Data from Experimental Studies (CAMARADES) checklist [46]. A 9point item list (each item received 1 point) was used to evaluate the risk of bias: (1) publication in a peer-reviewed journal; (2) statement describing animal temperature control; (3) randomization of treatment or control assignment; (4) allocation concealment; (5) blinded assessment of outcome; (6) avoidance of animal anesthetics with marked intrinsic properties; (7) sample size calculation; (8) statement of compliance with regulatory requirements; and (9) statement of possible conflict of interest.

2.6. Data Synthesis. We used Stata/SE 12.0 (Stata Corporation, College Station, TX, USA) to analyze the outcome data. The primary outcome was expressed as the standardized mean difference (SMD) and related 95\% confidence interval (CI). We then combined each outcome of interest across the included studies using the random-effects model. Heterogeneity was examined using Cochrane's $Q$-test $(p>$ 0.10 indicates significant heterogeneity) and the $I^{2}$ metric $\left(I^{2}<50 \%\right.$ indicates acceptable heterogeneity). Additionally, a subgroup analysis based on animal species, study design, disc degeneration model, or follow-up period was also performed to investigate the possible sources of heterogeneity in our results.

2.7. Sensitivity Analysis and Publication Bias Analysis. To evaluate the stability of the outcomes of the meta-analysis, a sensitivity analysis was performed. We also estimated the heterogeneity before and after omitting studies with obvious variance to identify potential sources of heterogeneity. In addition, a funnel plot was used to qualitatively assess the publication bias, and the Begg test and the Egger test were used to quantitatively assess the publication bias.

\section{Results}

3.1. Study Selection and Study Characteristics. The general process of literature retrieval and selection is shown in 
TABLE 1: Characteristics of the included studies.

\begin{tabular}{|c|c|c|c|c|c|c|c|c|}
\hline First author & $\begin{array}{c}\text { Publication } \\
\text { year }\end{array}$ & Country & $\begin{array}{l}\text { Manuscript } \\
\text { language }\end{array}$ & $\begin{array}{l}\text { Animal } \\
\text { species }\end{array}$ & $\begin{array}{c}\text { Disc } \\
\text { degeneration } \\
\text { model }\end{array}$ & $\begin{array}{l}\text { Study } \\
\text { design }\end{array}$ & $\begin{array}{l}\text { Observation } \\
\text { time (weeks) }\end{array}$ & PRP description \\
\hline Nagae et al. [36] & 2007 & Japan & English & Rabbit & $\begin{array}{c}\text { Nucleus } \\
\text { pulposus } \\
\text { aspiration }\end{array}$ & N-RCT & 8 & Autologous \\
\hline Sawamura et al. [26] & 2009 & Japan & English & Rabbit & $\begin{array}{l}\text { Nucleus } \\
\text { pulposus } \\
\text { aspiration }\end{array}$ & N-RCT & 8 & Autologous \\
\hline Chen et al. [29] & 2009 & China & English & Pig & $\begin{array}{l}\text { Chymopapain } \\
\text { induction }\end{array}$ & N-RCT & 8 & Allogenic \\
\hline Gullung et al. [34] & 2011 & USA & English & Rat & Needle puncture & RCT & 4 & Not available \\
\hline Hu et al. [40] & 2012 & China & Chinese & Rabbit & Needle puncture & RCT & 2 & Autologous \\
\hline Obata et al. [37] & 2012 & Japan & English & Rabbit & Needle puncture & N-RCT & 8 & Autologous \\
\hline Meng et al. [42] & 2013 & China & Chinese & Rabbit & Needle puncture & RCT & 8 & Allogenic \\
\hline Gui et al. [41] & 2015 & China & Chinese & Rabbit & Needle puncture & RCT & 2 & Autologous \\
\hline Gui et al. [33] & 2015 & China & English & Rabbit & Needle puncture & RCT & 6 & Autologous \\
\hline Wang et al. [38] & 2016 & China & English & Rabbit & Needle puncture & RCT & 8 & Allogenic \\
\hline Yang et al. [39] & 2016 & China & English & Rabbit & Needle puncture & RCT & 12 & Autologous \\
\hline
\end{tabular}

RCT: randomized controlled trial; N-RCT: nonrandomized controlled trial.

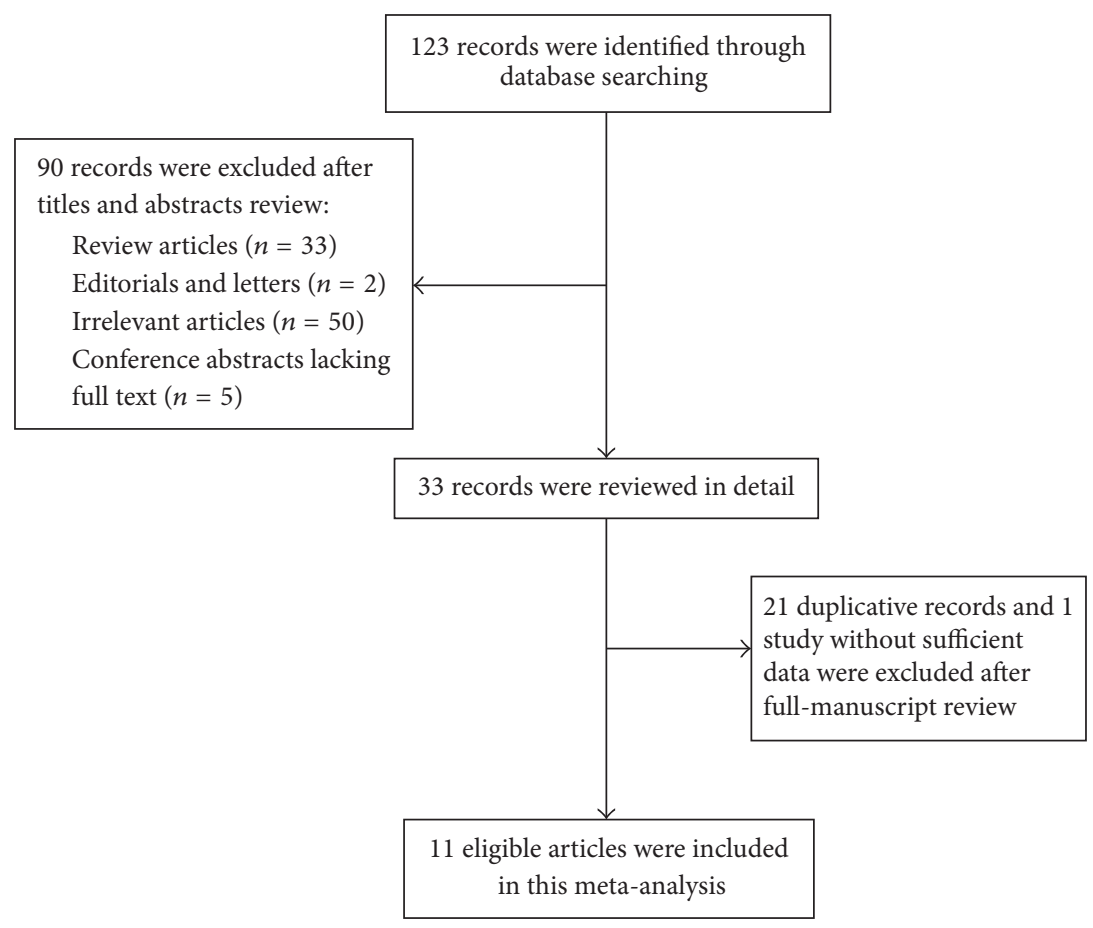

FIGURE 1: Flow diagram of the literature search and exclusion and inclusion process in the present meta-analysis.

Figure 1. Briefly, the database searches yielded 123 literatures. After title and abstract screening, 90 records that were review articles, editorials, letters, irrelevant articles, or conference abstracts were excluded. Thereafter, 21 duplicate studies and 1 study without sufficient data were excluded following fullmanuscript review. Finally, 11 studies were included in the present meta-analysis. Overall (as seen in Table 1), 419 discs were analyzed, of which 210 were treated with PRP and 209 were used as controls without PRP treatment. The animal species in the eligible studies included rabbit (9 studies, $81.82 \%)$, pig (1 study, 9.09\%), and rat (1 study, 9.09\%). Disc degeneration models in the eligible studies included needle puncture (8 studies, $72.73 \%$ ), nucleus pulposus aspiration (2 studies, $18.18 \%$ ), and chymopapain induction (1 study, 9.09\%). 
TABLE 2: Efficacy of PRP injection in retarding intervertebral disc degeneration.

\begin{tabular}{lcccccc}
\hline Outcomes & Number of studies & SMD & 95\% CI & $p$ value & $I^{2}$ & $\begin{array}{c}\text { Heterogeneity } p \\
\text { value }\end{array}$ \\
\hline Disc height & 7 & 2.66 & $1.86,3.47$ & 0.000 & $64.3 \%$ & 0.010 \\
T2 weighted MRI & 5 & -3.29 & $-4.44,-2.13$ & 0.000 & $68.9 \%$ & 0.012 \\
Histological degeneration grade & 3 & -4.28 & $-5.26,-3.30$ & 0.000 & $0.0 \%$ & 0.419 \\
Collagen II expression & 2 & 25389.74 & $-27585.72,78365.21$ & 0.348 & $93.7 \%$ & 0.000 \\
\hline
\end{tabular}

SMD: standardized mean difference; 95\% CI: 95\% confidence interval.

Study designs included RCT (7 studies 63.64\%) and N-RCT (4 studies, $36.36 \%$ ). The median final follow-up period was 8 weeks (range: $2-12$ weeks).

3.2. Literature Quality Assessment. Most of the studies in this meta-analysis did not perform a blinded assessment of the outcome and none of the studies included allocation concealment, a statement of animal temperature control, or sample size calculations. The quality assessment scores of the eligible studies ranged from 3 to 5 . Among the 11 eligible studies, 3 scored 5 points, 4 scored 4 points, and 4 scored 3 points. The mean quality score was 3.9 . The quality assessment of the eligible studies is summarized in Supplementary Table 1 (in Supplementary Material available online at https://doi.org/10.1155/2017/7919201).

3.3. Outcomes. As shown in Table 2, 7 studies evaluated the effects of PRP treatment on disc height. We found that disc height in the PRP-treated group was significantly higher than in the control group $(\mathrm{SMD}=2.66,95 \% \mathrm{CI}: 1.86$, $\left.3.47, p=0.000, I^{2}=64.3 \%\right)$. Five studies reported the effects of PRP treatment on MRI T2 signal intensity. The Pfirrmann classification score in the PRP treatment group was significantly lower than in the control group (SMD = $-3.29,95 \%$ CI: $\left.-4.44,-2.13, p=0.000, I^{2}=68.9 \%\right)$. In 3 studies, the histological degeneration grade was significantly lower in the PRP treatment than in the control group (SMD = $\left.-4.28,95 \% \mathrm{CI}:-5.26,-3.30, p=0.000, I^{2}=0.0 \%\right)$. However, no difference in the expression of collagen II between the PRP treatment group and the control group was found in the 2 eligible studies $(\mathrm{SMD}=25389.74,95 \% \mathrm{CI}:-27585.72$, 78365.21, $p=0.348, I^{2}=93.7 \%$ ) (the Forests plots were shown in Supplementary Figure 1).

3.4. Subgroup Analysis. Because heterogeneity was detected in the effects of PRP treatment on disc degeneration, a subgroup analysis of disc height and MRI T2 signal intensity by animal species, study design, disc degeneration model, follow-up period, and disc height measurement was performed to investigate the potential source of heterogeneity. Due to the limited eligible studies and low heterogeneity of the histological degeneration grade outcome, we did not perform a subgroup analysis for collagen II expression or histological degeneration grade. Overall, no obvious differences were found between our main analysis and the subgroup analysis (Tables 3 and 4).
3.5. Sensitivity Analysis and Publication Bias. In the sensitivity analysis for disc height outcome (Figure 2(a)), the initial heterogeneity $\left(I^{2}=64.3 \%\right.$, heterogeneity $p$ value $=$ $0.010)$ significantly decreased $\left(I^{2}=40.9 \%\right.$, heterogeneity $p$ value $=0.133$ ) when the study with obvious variance was removed (Gui et al. 2015, written in Chinese [41]). For the MRI T2 signal intensity outcome (Figure 2(b)), the initial heterogeneity $\left(I^{2}=68.9 \%\right.$, heterogeneity $p$ value $=$ 0.012 ) decreased when the study by Sawamura et al. [26] was removed $\left(I^{2}=47.5 \%\right.$, heterogeneity $p$ value $\left.=0.126\right)$. However, removal of this study did not dramatically alter the results of the overall pooled data for the effects of PRP treatment on disc height and MRI T2 signal intensity.

No obvious asymmetry in the outcomes of disc height and MRI T2 signal intensity was observed on the funnel plot by visual inspection (Figure 3 ). The Egger test ( $p=0.266$ and $p=0.399$ for disc height and MRI T2 signal, resp.) and the Begg test $(p=0.230$ and $p=0.462$ for disc height and MRI T2 signal, resp.) did not indicate any obvious publication bias. Because of the relatively limited number of studies on histological degeneration grade and collagen II expression outcomes, the publication bias analysis was not performed.

\section{Discussion}

PRP is a centrifuged blood product with high platelet content. Because it also contains a high level of growth factors, including TGF- $\beta 1$, IGF, and PDGF, PRP has been used to promote bone formation and soft-tissue repair in clinical practice $[47,48]$. Disc degeneration is regarded as a type of degenerative disease that is difficult to self-repair. Previously, several animal trials [26, 29, 33-42] have demonstrated that PRP treatment could inhibit the pathological process of intervertebral disc degeneration. However, the experimental parameters, including sample size, disc degeneration model induction method, follow-up period, and disc regeneration evaluation parameters, vary across studies. A quantitative analysis of synthesized homogeneous data may be helpful to overcome these shortcomings and to reach relatively reasonable conclusions. Therefore, we performed the present meta-analysis on the efficacy of PRP treatment in retarding disc degeneration in animal.

A degenerated disc undergoes serial pathological changes in its metabolism, biomechanics, geometric appearance, and cellular phenotype. These changes are characterized as reduced cell density, increased cell senescence and apoptosis, unbalanced matrix metabolism, upregulated inflammatory 
TABLE 3: Subgroup analysis of the disc height based on animal species, study design, disc degeneration model, follow-up period, and disc height measurement.

\begin{tabular}{|c|c|c|c|c|c|}
\hline Subgroup analysis index & Number of studies & SMD & $95 \% \mathrm{CI}$ & $p$ value & $I^{2}$ \\
\hline \multicolumn{6}{|l|}{ Animal species } \\
\hline Rabbit & 5 & 3.14 & $2.19,4.08$ & 0.000 & $62.8 \%$ \\
\hline Pig & 1 & 1.21 & $-0.04,2.45$ & 0.058 & NA \\
\hline Rat & 1 & 1.83 & $0.44,3.22$ & 0.010 & NA \\
\hline \multicolumn{6}{|l|}{ Study design } \\
\hline N-RCT & 3 & 2.50 & $0.91,4.09$ & 0.002 & $73.5 \%$ \\
\hline RCT & 4 & 2.82 & $1.75,3.89$ & 0.000 & $66.6 \%$ \\
\hline \multicolumn{6}{|l|}{ Disc degeneration model } \\
\hline Nucleus pulposus aspiration & 1 & 4.17 & $2.46,5.89$ & 0.000 & NA \\
\hline Chymopapain induction & 1 & 1.21 & $-0.04,2.45$ & 0.058 & NA \\
\hline Needle puncture & 5 & 2.68 & $1.86,3.51$ & 0.000 & $55.7 \%$ \\
\hline \multicolumn{6}{|l|}{ Follow-up period } \\
\hline 2 weeks & 1 & 6.01 & $3.58,8.44$ & 0.000 & NA \\
\hline 4 weeks & 1 & 1.83 & $0.44,3.22$ & 0.010 & NA \\
\hline 6 weeks & 1 & 2.67 & $1.55,3.80$ & 0.000 & NA \\
\hline 8 weeks & 4 & 2.43 & $1.49,3.37$ & 0.000 & $60.5 \%$ \\
\hline \multicolumn{6}{|l|}{ Disc height measurement } \\
\hline Absolute disc height & 2 & 2.95 & $0.66,5.23$ & 0.012 & $67.2 \%$ \\
\hline DHI\% & 5 & 2.59 & $1.65,3.53$ & 0.000 & $64.3 \%$ \\
\hline
\end{tabular}

RCT: randomized controlled trial; N-RCT: nonrandomized controlled trial. SMD: standardized mean difference; 95\% CI: 95\% confidence interval. DHI: disc height index.

TABLE 4: Subgroup analysis of T2 weighted MRI image based on animal species, study design, disc degeneration model, and follow-up period.

\begin{tabular}{|c|c|c|c|c|c|}
\hline Subgroup analysis index & Number of studies & SMD & $95 \%$ CI & $p$ value & $I^{2}$ \\
\hline \multicolumn{6}{|l|}{ Animal species } \\
\hline Rabbit & 5 & -3.29 & $-4.44,-2.13$ & 0.000 & $68.9 \%$ \\
\hline \multicolumn{6}{|l|}{ Study design } \\
\hline N-RCT & 1 & -1.85 & $-2.98,-0.73$ & 0.001 & NA \\
\hline RCT & 4 & -3.70 & $-4.45,-2.95$ & 0.000 & $47.5 \%$ \\
\hline \multicolumn{6}{|l|}{ Disc degeneration model } \\
\hline Nucleus pulposus aspiration & 1 & -1.85 & $-2.98,-0.73$ & 0.001 & NA \\
\hline Needle puncture & 4 & -3.70 & $-4.45,-2.95$ & 0.000 & $93.5 \%$ \\
\hline \multicolumn{6}{|l|}{ Follow-up period } \\
\hline 2 weeks & 2 & -3.04 & $-4.24,-1.83$ & 0.784 & $96.0 \%$ \\
\hline 6 weeks & 1 & -5.12 & $-6.83,-3.41$ & 0.000 & NA \\
\hline 8 weeks & 1 & -1.85 & $-2.98,-0.73$ & 0.001 & NA \\
\hline 12 weeks & 1 & -3.65 & $-4.81,-2.50$ & 0.000 & NA \\
\hline
\end{tabular}

RCT: randomized controlled trial; N-RCT: nonrandomized controlled trial. SMD: standardized mean difference; 95\% CI: 95\% confidence interval.

cytokines, and decreased disc height and NP hydration [1012, 49]. In this meta-analysis, we mainly investigated the effects of PRP treatment on four important disc regeneration parameters: alteration of disc height, MRI T2 signal intensity, histological degeneration grade, and collagen II expression. Notably, our main analysis demonstrated that PRP treatment could significantly increase disc height and MRI T2 signal intensity while reducing the histological degeneration grade (all $p$ value $<0.05$, Table 2 ). Consistent with this finding, the individual in vivo [26, 29, 33-42] and in vitro studies [2832] also demonstrated that PRP treatment has protective effects on the disc, such as promoting cell proliferation and matrix synthesis, decreasing cell apoptosis, increasing disc hydration, and increasing disc height. Together, our metaanalysis and these studies suggest that PRP treatment may be a promising therapy for retarding disc regeneration.

Because heterogeneity in disc height $\left(I^{2}=64.3 \%\right)$ and MRI T2 image $\left(I^{2}=68.9 \%\right)$ was observed in our main meta-analysis, a subgroup analysis to determine significantly sources of heterogeneity was performed. We did not conduct a subgroup analysis of the histological degeneration grade and collagen II expression due to the undetected heterogeneity $\left(I^{2}=0.0 \%\right)$ and limited number of eligible studies $(n=2)$, respectively. Generally, the subgroup analysis results 


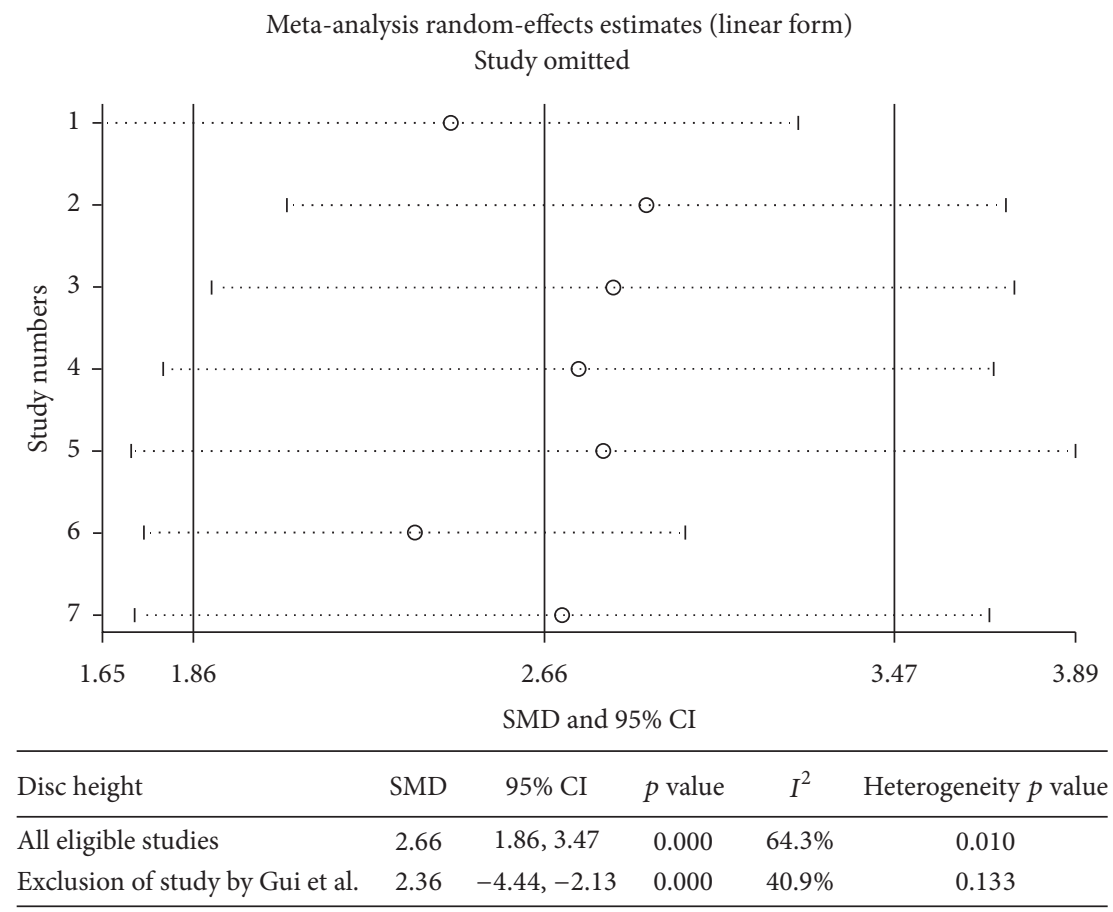

(a)

Meta-analysis random-effects estimates (linear form)

Study omitted

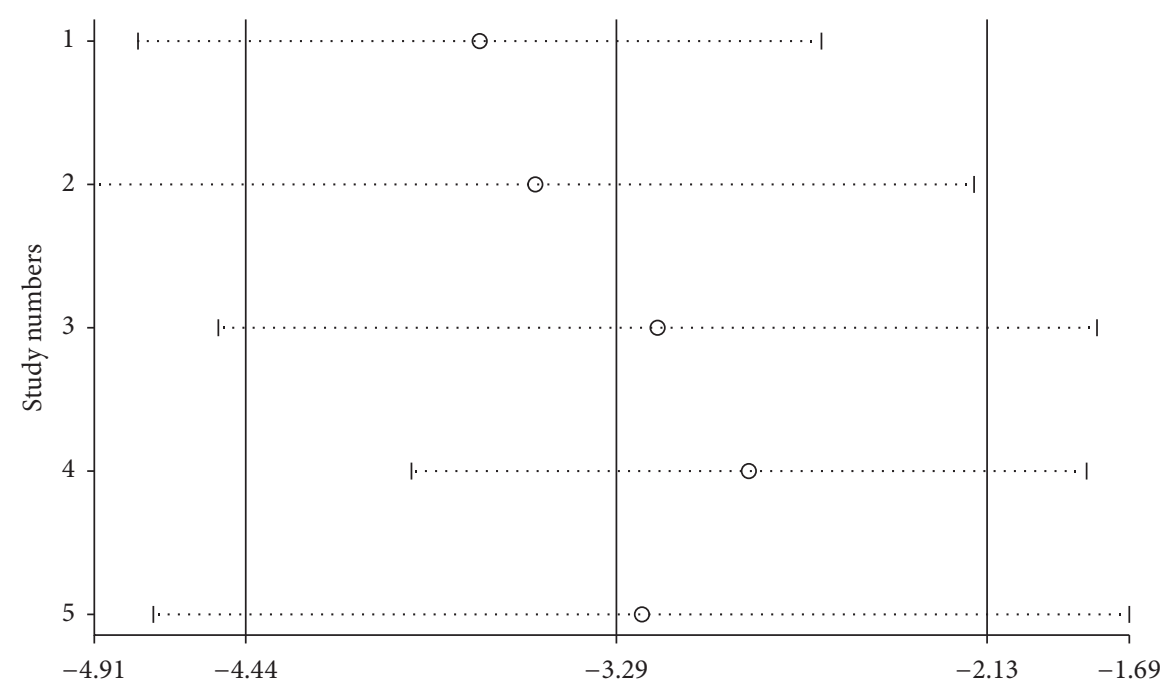

SMD and $95 \%$ CI

\begin{tabular}{lccccc}
\hline MRI T2 signal & SMD & 95\% CI & $p$ value & $I^{2}$ & Heterogeneity $p$ value \\
\hline All eligible studies & -3.29 & $-4.44,-2.13$ & 0.000 & $68.9 \%$ & 0.012 \\
Exclusion of study by Sawamura et al. & -3.70 & $-4.45,-2.95$ & 0.000 & $47.5 \%$ & 0.126 \\
\hline
\end{tabular}

(b)

FIGURE 2: Sensitivity analysis for disc height (a) and MRI T2 signal intensity (b). The three-line table ( $a$ and b) indicates the SMD, 95\% CI, and heterogeneity before and after the study with obvious variance was removed (SMD: standardized mean difference; 95\% CI: 95\% confidence interval). 
Begg's funnel plot with pseudo 95\% confidence limits

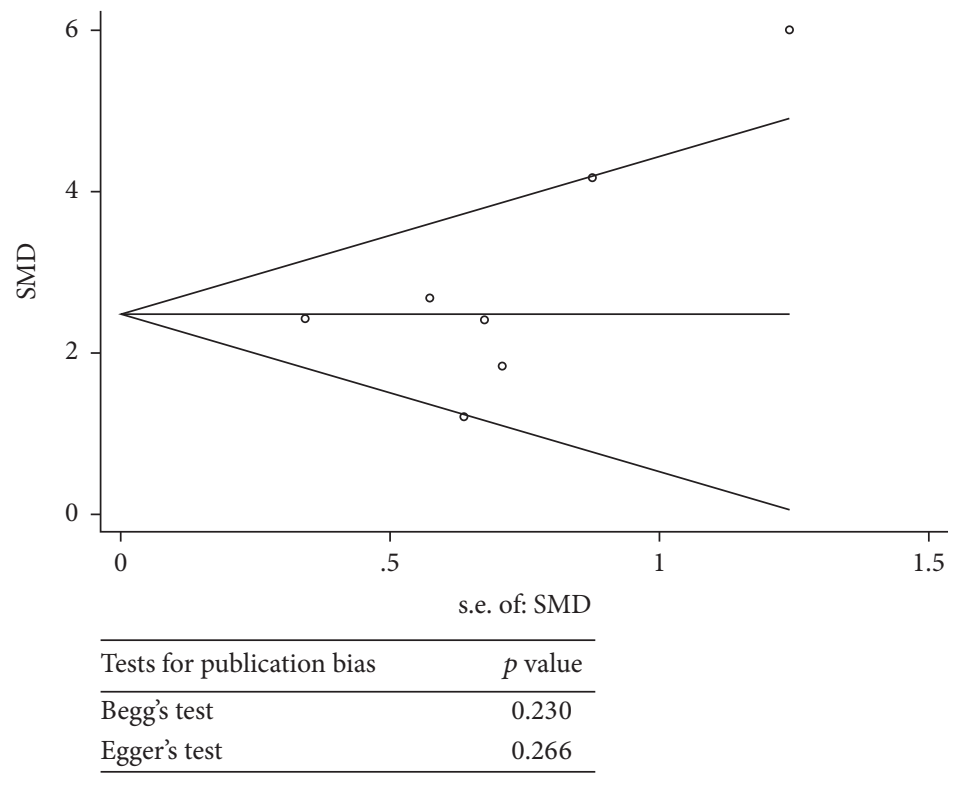

(a)

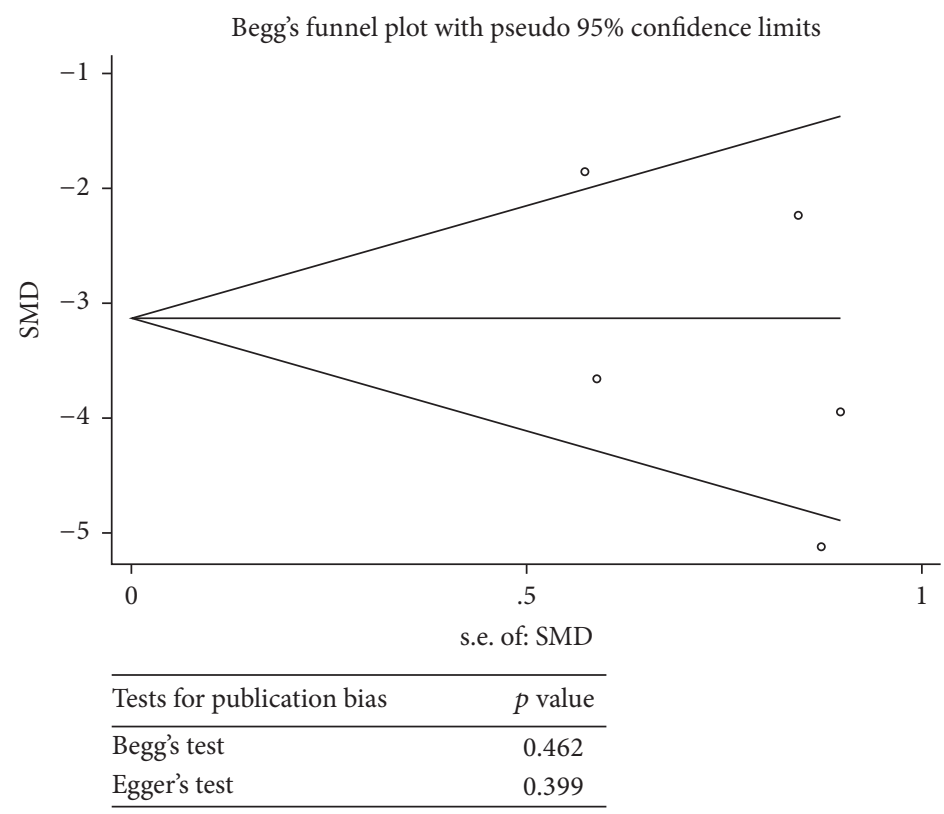

(b)

FIGURE 3: Funnel plot for the publication bias of studies on the effects of platelet-rich plasma (PRP) treatment on disc height (a) and MRI T2 signal intensity (b). The three-line table ( $\mathrm{a}$ and $\mathrm{b}$ ) indicates the $p$ value for the Begg test and the Egger test, respectively.

were consistent with those of the main analysis. However, the subgroup analysis of disc height outcome showed no significant difference in the disc height index in pig [29] or in the chymopapain-induced degeneration model after PRP treatment (all $p$ value $=0.058$, Table 3 ), which contradicts the significant difference found in the main analysis of disc height $(p=0.000)$. This indicates that the animal species and degeneration model induction method may be potential sources of heterogeneity for disc height outcome. Similarly, subgroup analysis of MRI T2 signal intensity indicated that follow-up period may be a potential source of heterogeneity for the T2 weighted MRI image. Based on these findings, it is possible that PRP treatment in large animals or in an animal degeneration model with heavy damage or with a short follow-up period does not exhibit statistically significant disc regeneration efficacy.

Sensitivity analysis is a common method for investigating study stability. Because the number of eligible studies for the histological degeneration grade and collagen II expression outcomes was low, we only performed the sensitivity analysis 
for studies that evaluated the efficacy of PRP treatment with respect to disc height restoration and TMRI T2 signal intensity. Our results showed that the heterogeneity in the disc height and TMRI T2 signal intensity outcomes decreased significantly when the study by Gui et al. [41] and the study by Sawamura et al. [26] were omitted (from 64.3\% to $40.9 \%$ and from $68.9 \%$ to $47.5 \%$, resp.). However, this did not dramatically alter the overall result of the pooled data with respect to changes in disc height and TMRI T2 signal intensity after PRP treatment. Moreover, no significant publication bias was detected for the disc height and MRI T2 signal intensity outcomes. These results imply that our metaanalysis results were stable. Additionally, these results indicate that certain underlying factors in the abovementioned studies may be a potential source for the heterogeneity of the synthesized data for changes in disc height and MRI T2 signal intensity. After screening the details of the 11 eligible studies, we speculate that the short follow-up period ( 2 weeks in the study by Gui et al. [41]) and the more exacerbated disc degeneration status (induction by NP aspiration in the study by Sawamura et al. [26]) are possible contributors to the decrease in heterogeneity when the corresponding studies were omitted.

This meta-analysis has several limitations. First, the accessible data of some of the eligible studies were relatively limited and not similar enough, which directly affected the cogency of our results. Second, because of our search strategy focused on English and Chinese articles, publication bias and selection bias were inevitable. Third, sensitivity analysis and publication bias analysis were not performed on the histological degeneration grade and collagen II expression outcomes due to the insufficient number of eligible studies. Fourth, when it comes to the molecular and cellular responses to regeneration, there are no objective criteria that truly identify intervertebral disc regeneration at the structural level. Because of these limitations, a more comprehensive literature-election process and an updated meta-analysis on the present topic that includes a greater number of eligible studies should be considered in the future.

\section{Conclusions}

This meta-analysis study demonstrated that PRP treatment plays an important role in disc regeneration, especially in restoring disc height of rodent rabbit and rat, reducing histological degeneration grade, and increasing MRI T2 signal intensity. This study provides direct theoretical support for the future application of PRP in retarding human disc degeneration.

\section{Conflicts of Interest}

The authors declared no conflicts of interest in this work.

\section{Acknowledgments}

The authors express thanks for the funding provided by the National Natural Science Foundation of China (81272029 and 81601932).

\section{References}

[1] S. Vedicherla and C. T. Buckley, "Cell-based therapies for intervertebral disc and cartilage regeneration- Current concepts, parallels, and perspectives," Journal of Orthopaedic Research, 2016.

[2] N. V. Vo, R. A. Hartman, P. R. Patil et al., "Molecular mechanisms of biological aging in intervertebral discs," Journal of Orthopaedic Research, vol. 34, no. 8, pp. 1289-1306, 2016.

[3] Z. Wang, C. M. Perez-Terzic, J. Smith et al., "Efficacy of intervertebral disc regeneration with stem cells - A systematic review and meta-analysis of animal controlled trials," Gene, vol. 564, no. 1, pp. 1-8, 2015.

[4] W. Wang, J. Hao, S. Zheng et al., "Association between cartilage intermediate layer protein and degeneration of intervertebral disc a meta-analysis," Spine, vol. 41, no. 20, pp. E1244-E1248, 2016.

[5] L. Nong, Y. Huang, S. Zhao, and N. Xu, "Vitamin D receptor gene, matrix metalloproteinase 3 polymorphisms and the risk of intervertebral disc degeneration susceptibility: Meta-analysis," Asian Spine Journal, vol. 10, no. 5, pp. 964-971, 2016.

[6] X. Deng, F. Zhao, B. Kang, and X. Zhang, "Elevated interleukin6 expression levels are associated with intervertebral disc degeneration," Experimental and Therapeutic Medicine, vol. 11, no. 4, pp. 1425-1432, 2016.

[7] G. Xu, Q. Mei, D. Zhou, J. Wu, and L. Han, "Vitamin D Receptor Gene and Aggrecan Gene Polymorphisms and the Risk of Intervertebral Disc Degeneration - A Meta-Analysis," PLoS ONE, vol. 7, no. 11, Article ID e50243, 2012.

[8] Z. Yan, L. Yin, Z. Wang et al., "A Novel Organ Culture Model of Mouse Intervertebral Disc Tissues," Cells Tissues Organs, vol. 201, no. 1, pp. 38-50, 2015.

[9] J. P. G. Urban and J. F. McMullin, "Swelling pressure of the intervertebral disc: Influence of proteoglycan and collagen contents," Biorheology, vol. 22, no. 2, pp. 145-157, 1985.

[10] T. Kadow, G. Sowa, N. Vo, and J. D. Kang, "Molecular Basis of Intervertebral Disc Degeneration and Herniations: What Are the Important Translational Questions?" Clinical Orthopaedics and Related Research, vol. 473, no. 6, pp. 1903-1912, 2015.

[11] P. Priyadarshani, Y. Li, and L. Yao, "Advances in biological therapy for nucleus pulposus regeneration," Osteoarthritis and Cartilage, vol. 24, no. 2, pp. 206-212, 2016.

[12] D. Sakai and S. Grad, "Advancing the cellular and molecular therapy for intervertebral disc disease," Advanced Drug Delivery Reviews, vol. 84, pp. 159-171, 2015.

[13] S. J. Lipson and H. Muir, "Experimental intervertebral disc degeneration: morphologic and proteoglycan changes over time," Arthritis and Rheumatism, vol. 24, no. 1, pp. 12-21, 1981.

[14] H. Cho, S. Lee, S.-H. Park, J. Huang, K. A. Hasty, and S.-J. Kim, "Synergistic effect of combined growth factors in porcine intervertebral disc degeneration," Connective Tissue Research, vol. 54, no. 3, pp. 181-186, 2013.

[15] A. J. Hayes and J. R. Ralphs, "The response of foetal annulus fibrosus cells to growth factors: modulation of matrix synthesis by TGF- $\beta 1$ and IGF-1," Histochemistry and Cell Biology, vol. 136, no. 2, pp. 163-175, 2011.

[16] H. Yang, C. Cao, C. Wu et al., “TGF- $\beta$ I Suppresses Inflammation in Cell Therapy for Intervertebral Disc Degeneration," Scientific Reports, vol. 5, Article ID 13254, 2015.

[17] J.-S. Kim, M. B. Ellman, H. S. An, A. J. Van Wijnen, J. A. Borgia, and H.-J. Im, "Insulin-like growth factor 1 synergizes with 
bone morphogenetic protein 7-mediated anabolism in bovine intervertebral disc cells," Arthritis and Rheumatism, vol. 62, no. 12, pp. 3706-3715, 2010.

[18] Z. Wang, C. Fu, Y. Chen et al., "FoxC2 enhances BMP7mediated anabolism in nucleus pulposus cells of the intervertebral disc," PLoS ONE, vol. 11, no. 1, Article ID e0147764, 2016.

[19] Y. Zhang, F. M. Phillips, E. J.-M. A. Thonar et al., "Cell therapy using articular chondrocytes overexpressing BMP-7 or BMP-10 in a rabbit disc organ culture model," Spine, vol. 33, no. 8, pp. 831-838, 2008.

[20] T. Chujo, H. S. An, K. Akeda et al., "Effects of growth differentiation factor- 5 on the intervertebral disc - In vitro bovine study and in vivo rabbit disc degeneration model study," Spine, vol. 31, no. 25, pp. 2909-2917, 2006.

[21] H. E. Gruber, G. L. Hoelscher, J. A. Ingram, S. Bethea, and E. N. Hanley, "IGF-1 rescues human intervertebral annulus cells from in vitro stress-induced premature senescence," Growth Factors, vol. 26, no. 4, pp. 220-225, 2008.

[22] F. Travascio, S. Elmasry, and S. Asfour, "Modeling the role of IGF-1 on extracellular matrix biosynthesis and cellularity in intervertebral disc," Journal of Biomechanics, vol. 47, no. 10, pp. 2269-2276, 2014.

[23] H. Pratsinis and D. Kletsas, "PDGF, bFGF and IGF-I stimulate the proliferation of intervertebral disc cells in vitro via the activation of the ERK and Akt signaling pathways," European Spine Journal, vol. 16, no. 11, pp. 1858-1866, 2007.

[24] H. E. Gruber, H. J. Norton, and E. N. Hanley Jr., "Anti-apoptotic effects of IGF-1 and PDGF on human intervertebral disc cells in vitro," Spine, vol. 25, no. 17, pp. 2153-2157, 2000.

[25] S. M. Presciutti, D. N. Paglia, T. Karukonda et al., "PDGF-BB inhibits intervertebral disc cell apoptosis in vitro," Journal of Orthopaedic Research, vol. 32, no. 9, pp. 1181-1188, 2014.

[26] K. Sawamura, T. Ikeda, M. Nagae et al., "Characterization of in vivo effects of platelet-rich plasma and biodegradable gelatin hydrogel microspheres on degenerated intervertebral discs," Tissue Engineering - Part A, vol. 15, no. 12, pp. 3719-3727, 2009.

[27] R. E. Marx, "Platelet-rich plasma (PRP): what is PRP and what is not PRP?” Implant Dentistry, vol. 10, no. 4, pp. 225-228, 2001.

[28] K. Akeda, H. S. An, R. Pichika et al., "Platelet-rich plasma (PRP) stimulates the extracellular matrix metabolism of porcine nucleus pulposus and anulus fibrosus cells cultured in alginate beads," Spine, vol. 31, no. 9, pp. 959-966, 2006.

[29] W. H. Chen, H. Y. Liu, W. C. Lo et al., "Intervertebral disc regeneration in an ex vivo culture system using mesenchymal stem cells and platelet-rich plasma," Biomaterials, vol. 30, no. 29, pp. 5523-5533, 2009.

[30] W.-H. Chen, W.-C. Lo, J.-J. Lee et al., “Tissue-engineered intervertebral disc and chondrogenesis using human nucleus pulposus regulated through TGF- $\beta 1$ in platelet-rich plasma," Journal of Cellular Physiology, vol. 209, no. 3, pp. 744-754, 2006.

[31] H. Cho, D. C. Holt, R. Smith, S.-J. Kim, R. J. Gardocki, and K. A. Hasty, "The Effects of Platelet-Rich Plasma on Halting the Progression in Porcine Intervertebral Disc Degeneration," Artificial Organs, vol. 40, no. 2, pp. 190-195, 2016.

[32] A. Mietsch, C. Neidlinger-Wilke, H. Schrezenmeier et al., "Evaluation of platelet-rich plasma and hydrostatic pressure regarding cell differentiation in nucleus pulposus tissue engineering," Journal of Tissue Engineering and Regenerative Medicine, vol. 7, no. 3, pp. 244-252, 2013.
[33] K. Gui, W. Ren, Y. Yu, X. Li, J. Dong, and W. Yin, "Inhibitory effects of platelet-rich plasma on intervertebral disc degeneration: A preclinical study in a rabbit model," Medical Science Monitor, vol. 21, pp. 1368-1375, 2015.

[34] G. B. Gullung, W. Woodall, M. Tucci, J. James, D. Black, and R. McGuire, "Platelet-rich plasma effects on degenerative disc disease: analysis of histology and imaging in an animal model," Evidence-Based Spine-Care Journal, vol. 2, no. 4, pp. 13-18, 2011.

[35] Y. Hou, G. Shi, J. Shi, G. Xu, Y. Guo, and P. Xu, "Study design: in vitro and in vivo assessment of bone morphogenic protein 2 combined with platelet-rich plasma on treatment of disc degeneration," International Orthopaedics, vol. 40, no. 6, pp. 1143-1155, 2016.

[36] M. Nagae, T. Ikeda, Y. Mikami et al., "Intervertebral disc regeneration using platelet-rich plasma and biodegradable gelatin hydrogel microspheres," Tissue Engineering, vol. 13, no. 1, pp. 147-158, 2007.

[37] S. Obata, K. Akeda, T. Imanishi et al., "Effect of autologous platelet-rich plasma-releasate on intervertebral disc degeneration in the rabbit anular puncture model: A preclinical study," Arthritis Research and Therapy, vol. 14, no. 6, article no. R241, 2012.

[38] S.-Z. Wang, J.-Y. Jin, Y.-D. Guo et al., "Intervertebral disc regeneration using platelet-rich plasma-containing bone marrowderived mesenchymal stem cells: A preliminary investigation," Molecular Medicine Reports, vol. 13, no. 4, pp. 3475-3481, 2016.

[39] H. Yang, C. Yuan, C. Wu et al., "The role of TGF- $\beta 1 / \mathrm{Smad} 2 / 3$ pathway in platelet-rich plasma in retarding intervertebral disc degeneration," Journal of Cellular and Molecular Medicine, vol. 20, no. 8, pp. 1542-1549, 2016.

[40] X. Hu, C. Wang, and Y. Rui, "An experimental study on effect of autologous platelet-rich plasma on treatment of early intervertebral disc degeneration," Chinese Journal of Reparative and Reconstructive Surgery, vol. 26, no. 8, pp. 977-983, 2012.

[41] K. Gui, Y. Yu, W. Ren, X. Li, J. Dong, and W. Yin, "Interentional effect of platelet-rich plasma (PRP) on a rabbit model of earlystage intervertebral disc degeneration (IDD)," Fudan University Journal of Medical Sciences, vol. 42, pp. 204-211, 2015.

[42] F.-X. Meng, F. Li, C.-Q. Ye, Y.-B. Yin, and Y. Gao, "Adiposederived mesenchymal stem cells combined with platelet gel for repair of intervertebral disc degeneration in rats," Chinese Journal of Tissue Engineering Research, vol. 17, no. 21, pp. 38013808, 2013.

[43] D. Moher, A. Liberati, J. Tetzlaff, and D. G. Altman, "Preferred reporting items for systematic reviews and meta-analyses: the PRISMA statement," International Journal of Surgery, vol. 8, no. 5, pp. 336-341, 2010.

[44] C. W. A. Pfirmann, A. Metzdorf, M. Zanetti, J. Hodler, and N. Boos, "Magnetic resonance classification of lumbar intervertebral disc degeneration," Spine, vol. 26, no. 17, pp. 18731878, 2001.

[45] T. Nomura, J. Mochida, M. Okuma, K. Nishimura, and K. Sakabe, "Nucleus pulposus allograft retards intervertebral disc degeneration," Clinical Orthopaedics and Related Research, no. 389, pp. 94-101, 2001.

[46] M. R. Macleod, T. O’Collins, D. W. Howells, and G. A. Donnan, "Pooling of animal experimental data reveals influence of study design and publication bias," Stroke, vol. 35, no. 5, pp. 1203-1208, 2004.

[47] G. Crovetti, G. Martinelli, M. Issi et al., "Platelet gel for healing cutaneous chronic wounds," Transfusion and Apheresis Science, vol. 30, no. 2, pp. 145-151, 2004. 
[48] E. Anitua, I. Andia, B. Ardanza, P. Nurden, and A. T. Nurden, "Autologous platelets as a source of proteins for healing and tissue regeneration," Thrombosis and Haemostasis, vol. 91, no. 1, pp. 4-15, 2004.

[49] S. P. F. Hughes, A. J. Freemont, D. W. L. Hukins, A. H. McGregor, and S. Roberts, "The pathogenesis of degeneration of the intervertebral disc and emerging therapies in the management of back pain," Journal of Bone and Joint Surgery - Series B, vol. 94, no. 10, pp. 1298-1304, 2012. 


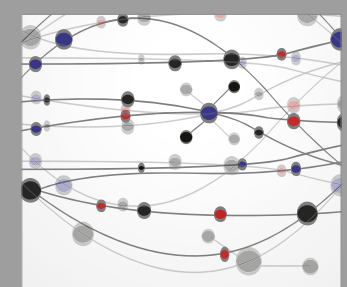

The Scientific World Journal
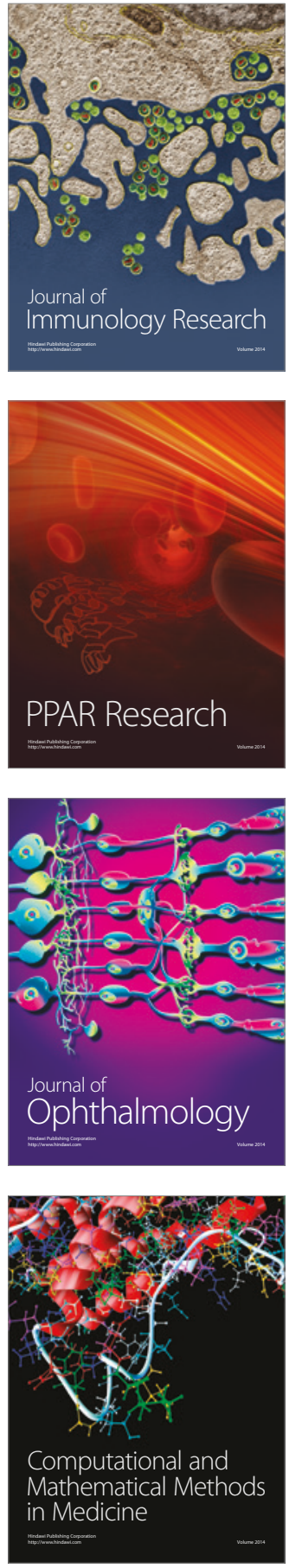

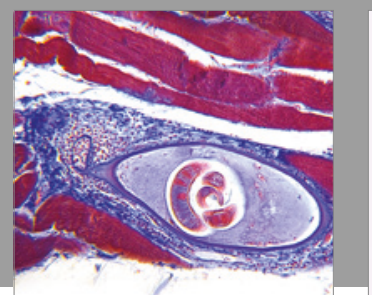

Gastroenterology Research and Practice
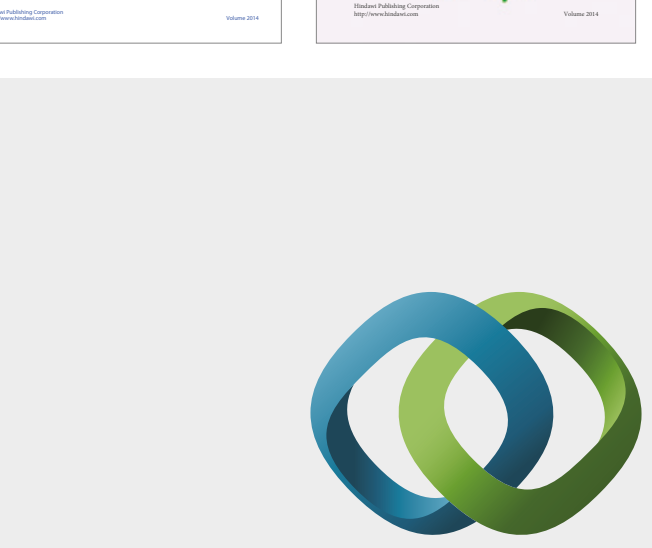

\section{Hindawi}

Submit your manuscripts at

https://www.hindawi.com
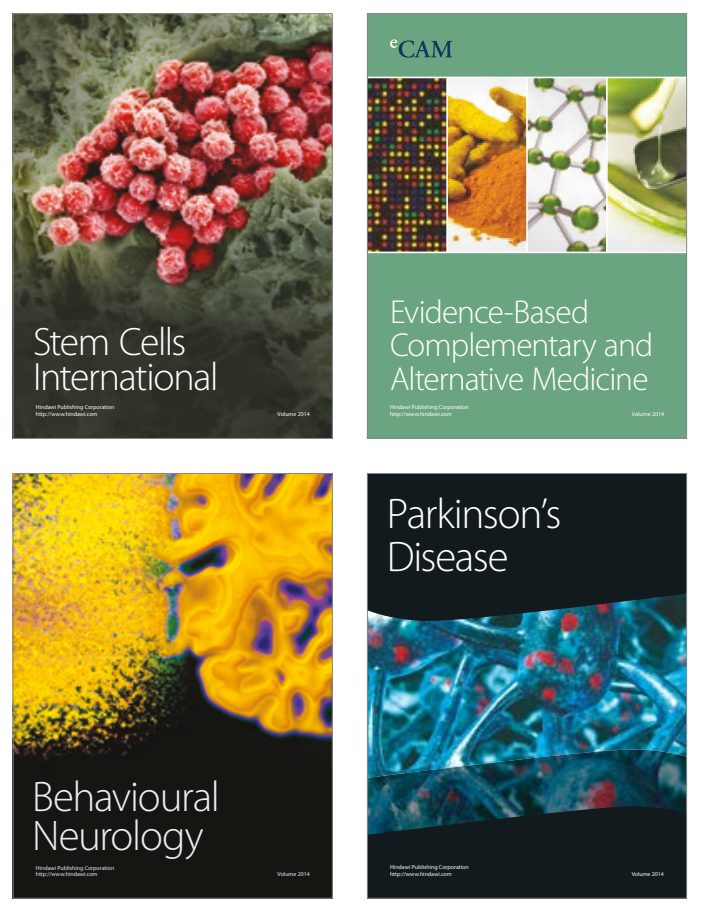
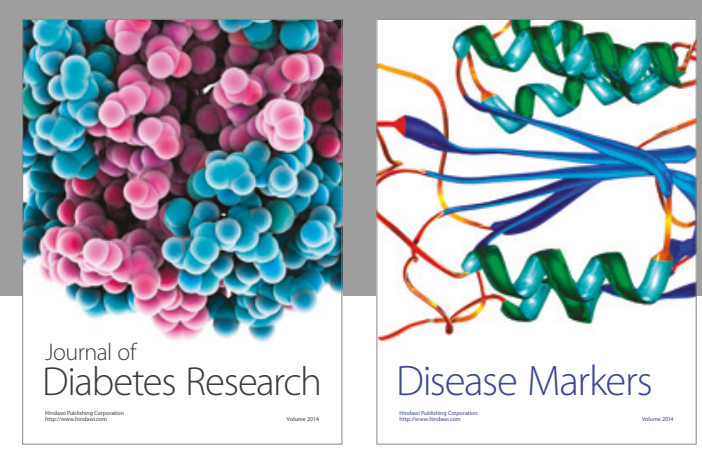

Disease Markers
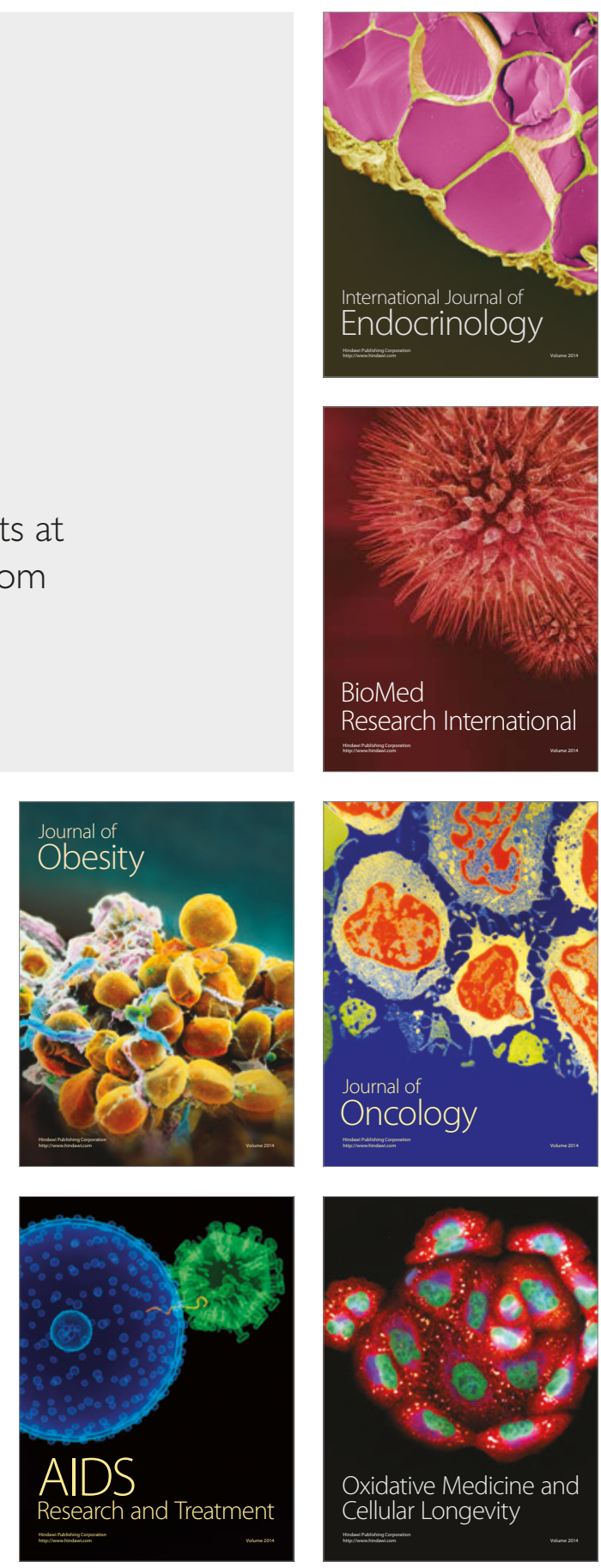